The Astrophysical Journal, 435:L31-L34, 1994 November 1

(C) 1994. The American Astronomical Society. All rights reserved. Printed in U.S.A.

\title{
FIRST HUBBLE SPACE TELESCOPE OBSERVATIONS OF THE BRIGHTEST STARS IN THE VIRGO GALAXY M100 $=$ NGC $4321^{1}$
}

\author{
Wendy L. Freedman, ${ }^{2}$ Barry F. Madore, ${ }^{3}$ Peter B. Stetson, ${ }^{4}$ Shaun M. G. Hughes, ${ }^{5}$ Jon A. Holtzman, ${ }^{6}$ \\ Jeremy R. Mould, ${ }^{7}$ John T. Trauger ${ }^{8}$ John S. Gallagher III, ${ }^{9}$ Gilda E. Ballester, ${ }^{10}$ \\ Christopher J. Burrows, ${ }^{11}$ Stefano Casertano, ${ }^{12}$ John T. Clarke, ${ }^{10}$ David Crisp, 5 \\ Laura Ferrarese, ${ }^{12}$ Holland Ford, ${ }^{11}$ J. A. Graham, ${ }^{13}$ Richard E. Griffiths, ${ }^{12}$ \\ J. JefF Hester, ${ }^{14}$ Robert Hill, ${ }^{2}$ John G. Hoessel, ${ }^{9}$ John Huchra, ${ }^{15}$ \\ Robert C. Kennicutt, ${ }^{16}$ Paul A. Scowen, ${ }^{14}$ Bill Sparks, ${ }^{11}$ \\ Karl R. Stapelfeldt, ${ }^{8}$ Alan M. Watson, ${ }^{9}$ \\ AND JIM WESTPHAL ${ }^{17}$ \\ Received 1994 May 17; accepted 1994 August 17
}

\begin{abstract}
As part of both the Early Release Observations from the Hubble Space Telescope and the Key Project on the Extragalactic Distance Scale, we have obtained multiwavelength BVR WFPC2 images for the face-on Virgo cluster spiral galaxy $\mathrm{M} 100=$ NGC 4321 . We report here preliminary results from those observations, in the form of a color-magnitude diagram for $\sim 11,500$ stars down to $V \sim 27$ mag and a luminosity function for the brightest blue stars which is found to have a slope of 0.7 , in excellent agreement with previous results obtained for significantly nearer galaxies. With the increased resolution now available using WFPC2, the number of galaxies in which we can directly measure Population I stars and thereby quantify the recent evolution, as well as test stellar evolution theory, has dramatically increased by at least a factor of 100 . Finally, we find that stars are present in M100 at the colors and luminosities expected for the brightest Cepheid variables in galaxies.
\end{abstract}

Subject headings: galaxies: distances and redshifts - galaxies: individual (M100, NGC 4321) - Cepheids stars: early type

\section{INTRODUCTION}

$\mathrm{M} 100(=\mathrm{NGC} 4321)$ is a luminous spiral galaxy in the Virgo cluster that is seen nearly face-on, with an apparent axial ratio of 0.87 ; Tully 1988). Having very well defined symmetric spiral arms, it is classified by Sandage \& Tammann (1981) as Sc(s) I

\footnotetext{
${ }^{1}$ Based on observations with the NASA/ESA Hubble Space Telescope, obtained at the Space Telescope Science Institute, which is operated by AURA, Inc. under NASA Contract No. NAS 5-26555.

${ }^{2}$ The Observatories, Carnegie Institution of Washington, Pasadena, CA 91101.

${ }^{3}$ NASA/IPAC Extragalactic Database, Infrared Processing and Analysis Center, California Institute of Technology, Pasadena, CA 91125.

4 Dominion Astrophysical Observatory, 5071 West Saanich Road, Victoria, BC, Canada, V8X 4M6. UK.

${ }_{5}^{5}$ Royal Greenwich Observatory, Madingley Road, Cambridge, CB3 0EZ,

${ }^{6}$ Lowell Observatory, Mars Hill Road, Flagstaff, AZ 86001.

${ }^{7}$ MSSO, Weston, P.O., ACT 2611, Australia.

8 Jet Propulsion Laboratory, 4800 Oak Grove Drive, Mail Stop 169-237, Pasadena, CA 91109.

${ }^{9}$ University of Wisconsin, Madison, Department of Astronomy, 475 North Charter Street, Madison, WI 53706.

10 University of Michigan, Department of Astronomy, Dennison Building, Ann Arbor, MI 48109-1090.

${ }^{11}$ Space Telescope Science Institute, 3700 San Martin Drive, Baltimore, MD 21218.

${ }^{12}$ Department of Physics and Astronomy, Bloomberg 501, Johns Hopkins University, 3400 North Charles Street, Baltimore, MD 21218.

${ }^{13}$ Department of Terrestrial Magnetism, Carnegie Institution öf Washington, 5241 Broad Branch Road NW, Washington, DC 20015.

14 Arizona State University, Department of Physics and Astronomy, Tyler Mall, Tempe, AZ 85287.

${ }^{15}$ Harvard College, Center for Astrophysics, 60 Garden Street, Cambridge, MA 02138.

${ }_{16}$ Steward Observatory, University of Arizona, Tucson, AZ 85721.

${ }^{17}$ California Institute of Technology, Pasadena, CA 91125.
}

and by de Vaucouleurs et al. (1976) as SAB(s)bc. From the ground this galaxy is not well resolved, and little was previously known of its stellar populations.

As part of the Early Release Observation program associated with the initial commissioning phases of WFPC2, M100 was imaged in three filters and at two epochs. The purpose of these observations was to provide both a test of the corrective optics and a feasibility study of Virgo cluster observations for the Extragalactic Distance Scale Key Project. The Key Project was begun in 1992 December using WF/PC (WFPC1) to observe the nearby galaxy M81; a distance to that galaxy based on the HST discovery of 30 new Cepheids has recently been published by Freedman et al. (1994). The calibration procedure and a discussion of the stellar populations was given in Hughes et al. (1994). The primary aim of the Key Project is to measure Cepheid distances to about two dozen nearby galaxies useful for calibrating a number of secondary distance methods. In addition, the Key Project target list includes four galaxies in the Virgo cluster, one of which is M100.

A well-determined distance to the Virgo cluster is important for many reasons. Due both to its proximity and the fact that it contains a wide range of galaxy types, a large number of secondary-distance techniques have been applied to this cluster. The distance to the Virgo cluster has been a focal point for discussion because published values have spanned a wide range: the "long" and the "short" estimates of the extragalactic distance scale. It is now generally agreed that the distances to galaxies within the general vicinity of the Local Group (out to $\sim 1 \mathrm{Mpc}$ ) have been obtained to an accuracy and precision of $\pm 10 \%$ (Fukugita, Hogan, \& Peebles 1993). However, current estimates for the distance to Virgo range from $\sim 15-21 \mathrm{Mpc}$ (e.g., see the review by Jacoby et al. 1992; 
but also see Sandage 1993, who finds a value of $27 \mathrm{Mpc}$ for the distance to M100). These estimates differ by the uncertainty commonly associated with the far-field value of the Hubble constant itself.

The core of the Virgo cluster is devoid of spiral galaxies; hence the most appropriate target in Virgo is M100, which is $4^{\circ}$ NW of M87. The Virgo cluster is thought to extend from 10 to $18 \mathrm{Mpc}$ (Tonry, Ajhar, \& Luppino 1990), and further in Virgo South. Nevertheless, a distance to a Virgo galaxy is a critical test of the competing distance scales. Virgo is located $\sim 16$ Mpc away according to the short distance scale; hence 20 day Cepheids in Virgo would be predicted to have $\langle V\rangle=26 \mathrm{mag}$. If Virgo is located at the long distance of $\sim 27 \mathrm{Mpc}$ (Sandage 1993), the 20 day Cepheids are predicted to be more than 1 mag fainter. For these reasons, we have placed high priority on observing M100.

In the long term, direct Cepheid distances to a significant sample of Virgo galaxies will settle the controversy over the short and long distance scales for the cluster itself: a vital step, since it is currently the uncertainty in the distance rather than the velocity, that plays the critical role in the present controversy over the value of the Hubble constant. Moreover, both the Virgo and Fornax clusters have an important role to play in calibrating secondary distance indicators which tie the local distance measurements to the global expansion.

Before the repair of the optics for $H S T$, resolved studies of the most luminous stars in spiral galaxies were confined primarily to those within the Local Group or within nearby groups such as those associated with M81 and M101. (For a review of studies of luminous supergiants in external galaxies, see, for example, Humphreys 1987; Cepheid studies have been reviewed by Madore \& Freedman 1991 and Jacoby et al. 1992). Recently, some ground-based measurements of the brightest objects in Virgo galaxies have been carried out under excellent seeing conditions $\left(\sim 00^{\prime \prime} 4-00^{\prime \prime} 6\right)$ by Shanks et al. (1991), and Pierce, McClure, \& Racine (1992). With the wide-field camera/ planetary camera the effective resolution at Virgo is 5 times/10 times higher than can presently be achieved from the ground, and is now comparable to observing M81 from the ground at $\sim 0$. $5 / \sim 0$ ".25 resolution, or observing typical Local Group galaxies with $\sim 2^{\prime \prime} / \sim 1^{\prime \prime}$ resolution.

In this Letter we present color-magnitude diagrams and luminosity functions for the brightest objects in M100. In addition to the brightest stars, we identify several young clusters and inner regions of associations in the spiral arms. Finally, the feasibility of discovering Cepheids in this galaxy using HST is discussed. The M100 nucleus is the subject of another paper by Gallagher et al. (1994); photometry of the clusters will be presented by Madore et al. (1994).

\section{THE OBSERVATIONS, REDUCTIONS, AND CALIBRATION}

Data were obtained with WFPC2 at two separate epochs separated by 7 days: 1993 December 31 and 1994 January 7 .

- The F555W $(V)$ images for the three WFC chips are shown in Figure 1 (Plate L19). Exposure times were as follows: F439W $(4 \times 900 \mathrm{~s})$, F555W $(3 \times 900 \mathrm{~s}$ and $769 \mathrm{~s})$, F702W $(5 \times 600 \mathrm{~s}$ and 519 s). The latter exposures at F555W and F702W were cut short because of a loss of lock on the guide stars.

The M100 frames were processed using calibration data from the WFPC2 ground thermal-vacuum (TV) test. The initial processing of the individual raw frames consisted of a very small $A / D$ correction, subtraction of a bias level and superbias frame, subtraction of a superdark frame, and a tiny shutter shading correction. Subsequently, the frames in each filter were averaged and cosmic rays were rejected. From subsequent on-orbit flats, the flat-fielding used for the M100 frames is estimated to be good to a few percent rms, with peak errors of $\sim 10 \%$ in the corners of each chip.

An initial pass at the photometry of the averaged, cosmicray-cleaned frames was made using the aperture photometry routine available in the software package DAOPHOT II (e.g., Stetson 1992 and references therein). Later, profile-fitting photometry was obtained (as described below) on the original, unaveraged frames. A comparison of the aperture photometry between the averaged and unaveraged frames yielded differences amounting to less than $1 \%$. Profile-fitting photometry of the original frames was performed using DAOPHOT II and ALLFRAME (Stetson 1994a, b).

The frame-to-frame repeatability of stars measured in WFPC2 images - both those of M100 and of $\omega$ Centauri, as well as numerous simulated images - was found to be $\sim 0.03$ mag per exposure for stars from just below the magnitude level of incipient saturation to a magnitude level $\sim 4$ mag fainter. Below that, the errors increased in reasonable agreement with what would be expected from photon statistics. External errors will be larger and cannot be estimated from our repeat observations since the field always fell identically on the chips. A further source of uncertainty comes from the recently discovered problem with charge-transfer efficiency. ${ }^{18}$ For these reasons, the photometry presented in this Letter should be considered as preliminary only.

The transformation from the instrumental (F439W, F555W, F702W) to the standard Johnson-Kron-Cousins BVR system was accomplished in two steps. First, using data for stars in the field of the globular cluster $\omega$ Cen, a transformation was made from the WPFC2 instrumental magnitude system to the WFPC1 system, as defined by Harris et al. (1993). Details of this calibration procedure undertaken by the WFPC2 team are given by Holtzman et al. (1994). Finally, the transformations given by Harris et al. (1991) were used to transform to the standard BVR system.

\section{COLOR-MAGNITUDE DIAGRAMS AND LUMINOSITY FUNCTION}

ALLFRAME photometry was obtained for a total of 30,551 stars and starlike objects in the three WFC fields, and a further 1580 objects in the PC field. Stringent CHI restrictions were placed on the data to minimize the contamination by clearly resolved, nonstellar objects. ${ }^{19}$ The resulting $V$ versus $(B-V)$ color-magnitude diagram for the WFC chip 3 is shown in Figure 2. Plotted are $\sim 11,500$ stars with ALLFRAME

\footnotetext{
18 Subsequent to these observations, it was discovered that there are charge-transfer problems with the WFPC2 chips at the operating temperature used for these observations $\left(-77^{\circ} \mathrm{C}\right)$. This charge-transfer problem leads to variations in effective sensitivity depending on the row number in which an object falls. The effect gives a gradient of $\sim 10 \%$ in the photometry for the calibration data used here, but is possibly reduced in the M100 data which has a significant background (from the light of unresolved stars). Because of this effect, as well as scatter in the flight and ground observations of the calibration fields, the photometric zeropoints could be off by several percent $(5 \%-10 \%)$ and there is possibly a gradient in the photometry across the M100 frames leading to an effective additional rms error of $\sim 3 \%-5 \%$ when objects over the entire field are considered.

19 For readers who are not DAOPHOT afficionados, CHI is the ratio of observed to expected rms residual. If the noise model is correct, the average value of $\mathrm{CHI}$ should be close to unity; any object with $\mathrm{CHI} \gg 1$ is probably nonstellar.
} 


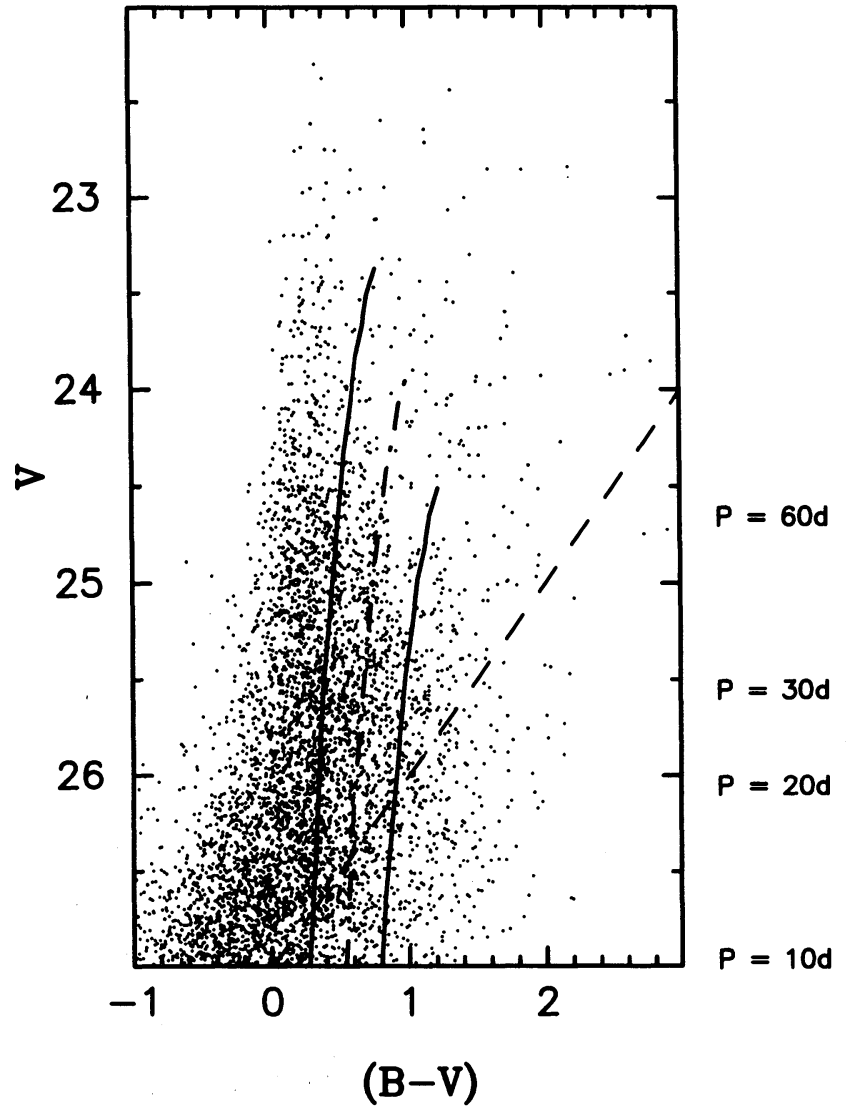

Fig. 2. $-V$ vs. $(B-V)$ color-magnitude diagram for $\sim 11,500$ stars measured by ALLFRAME on WFC2 chip 3. The solid lines indicate the position of the unreddened mean Cepheid instability strip for a distance modulus of $31.3 \mathrm{mag}(18 \mathrm{Mpc})$. The corresponding magnitude levels appropriate for this distance modulus for periods of $10,20,40$, and 60 days are labeled. The dashed line corresponds to $B=27 \mathrm{mag}$.

$\mathrm{CHI}<1.4$. About $12 \%$ of the total objects measured were eliminated on the basis of this stringent $\mathrm{CHI}$ limit.

The brightest blue supergiants in the color-magnitude diagram have $V \sim 22.5, B-V \sim 0.2 \mathrm{mag}$. Present also are red supergiants with $V \sim 22.8, B-V \sim 1.5 \mathrm{mag}$. The solid lines represent the mean Cepheid instability strip (described below). Fainter than $V=22$ mag contamination by foreground Galactic field stars is negligible in the 1.8 square arcmin area of a single WFC2 chip (Bahcall \& Soneira 1981; Ratnatunga \& Bahcall 1985); therefore, essentially all of the measured sources contributing to Figure 2 belong to M100.

Stars with $\mathrm{CHI}>1.4$ were flagged as potential star cluster candidates. A plot of $\mathrm{CHI}$ versus magnitude revealed that over the range of the brightest $2 \mathrm{mag}$, almost all objects had large $\mathrm{CHI}$ values $(>1.4)$. Most of these clusters are very blue $(B-V \sim 0.2 \mathrm{mag})$ and are thus young. Many of these objects have FWHM intensity-diameters of $<0$ ".5 and thus would appear to be stellar in most images taken with existing groundbased telescopes. Interestingly, there appears to be a ringlike concentration of compact star clusters around the periphery of the nuclear disk. These clusters will be discussed in more detail in a future paper (Madore et al. 1994).

A luminosity function for the blue supergiants is shown in Figure 3. A line of slope 0.7 (the mean slope determined by Freedman (1985) for a sample of 10 nearby spiral and irregular galaxies) is superposed on the M100 data; the agreement is

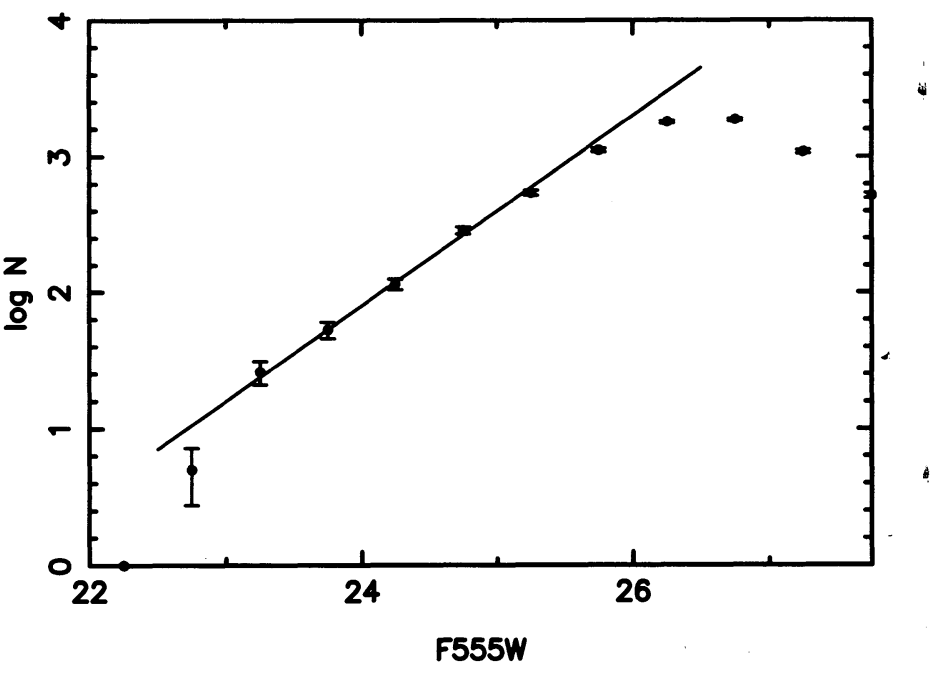

FIG. 3. $-V$-band luminosity function for the brightest blue stars in $\mathbf{M} 100$

excellent. This result extends and confirms earlier work indicating that, to within the uncertainties, the slope of the upper end of the luminosity function appears to be universal for galaxies with a wide range of morphological types and metallicities.

In Figure 2 the Cepheid instability strip (Madore \& Freedman 1991) is plotted after having been shifted by $31.3 \mathrm{mag}$ in apparent distance modulus (an illustrative mean value of the Virgo modulus, based on measurements of seven independent secondary distance indicators tabulated by Jacoby et al. 1992). In Table 1 , the expected (unreddened) $V$ magnitudes for Cepheids of 20,40, and 60 days are given for apparent distance moduli of 30.9 and $31.5 \mathrm{mag}$, based on the above-cited periodluminosity calibration. These distance moduli correspond to 15 and $20 \mathrm{Mpc}$, respectively. A 40 day Cepheid will have $\langle V\rangle \sim 25.1 \mathrm{mag}$ if Virgo is at the closer distance and $\langle V\rangle \sim 25.7 \mathrm{mag}$ if Virgo is at the farther distance. If M100 is as distant as $27 \mathrm{Mpc}$ or $\mu \sim=32.2 \mathrm{mag}$ ), the 60 day Cepheids would still have $\langle V\rangle \sim 25.9 \mathrm{mag}$, a magnitude limit well within the reach of WFPC2 (see Fig. 2).

The exposure times for the Early Release Observations at each individual epoch were short (in total less than $1800 \mathrm{~s}$ at $V$ and $1200 \mathrm{~s}$ at $R$ ). Hence an improvement in signal-to-noise ratio can be achieved with longer exposures. Moreover, the effects of crowding can be decreased by working at a lower surface brightness level at greater radial distance from the nucleus. Finally, the PC can be used to full advantage (with its increased sampling of the PSF) by positioning it away from the nucleus in an active star-forming region where Cepheids are expected to be located. Based on these preliminary data we conclude that the discovery of Cepheid variables in M100 is

TABLE 1

UNREDDENED CEPHEID $V$ MAGNITUdeS as a Function of Distance

\begin{tabular}{lcc}
\hline \hline $\begin{array}{c}\text { Period } \\
\text { (days) }\end{array}$ & $\begin{array}{c}15 \mathrm{Mpc}(\mu=30.9) \\
(V \text { magnitude })\end{array}$ & $\begin{array}{c}\text { 20 Mpc }(\mu=31.5) \\
(V \text { magnitude })\end{array}$ \\
\hline $20 \ldots \ldots$ & 25.9 & 26.5 \\
$40 \ldots \ldots$ & 25.1 & 25.7 \\
$60 \ldots \ldots$ & 24.6 & 25.2 \\
\hline
\end{tabular}


feasible using WFPC2, and our scheduled Key Project observations (optimized as described above) are now underway.

The referee has suggested that some readers may be tempted to estimate a distance to M100 using the brightest red and blue supergiants found in Figure 2. In the case of the red supergiants, for example, there are two groups of red stars, one at $V \sim 22.8 \mathrm{mag}$ and another at 23.7-23.9 mag. The choice of which group represents the brightest red stars would result in very different distance moduli (30.8 or $31.8 \mathrm{mag}$, respectively, assuming $M_{V} \sim-8.0 \mathrm{mag}$ ). However, neither of these distance estimates is robust. First, our data do not cover the entire galaxy, leaving open the possibility that the brightest supergiants are not within the field surveyed. Second, and most important, image crowding may be a serious problem and must be quantitatively addressed before we can have confidence in the magnitudes of the brightest stars. For example, the magnitudes of the brightest blue supergiants measured using an aperture of radius 2 pixels $(0.2)$ are over 1 full magnitude brighter than those measured using ALLFRAME and imposing a very strict $\mathrm{CHI}$ criterion as discussed above. Therefore we prefer to proceed and measure a distance to M100 using Cepheid variables, and then check whether the magnitudes of the brightest measured supergiants are consistent with current supergiant calibrations.

\section{SUMMARY}

Above all, the results of this Letter demonstrate the impressive capability of $H S T$ for studying the resolved stellar populations in galaxies well beyond the Local Group. $B V R$ color-magnitude diagrams and a luminosity function for the brightest blue supergiants have been measured in a galaxy at a distance representative of the Virgo cluster. These results demonstrate that we now have the potential to study the rich variety of galaxies present in the Virgo cluster with comparable resolution to that of several galaxies in our own Local Group; moreover, for galaxies closer than those in the Virgo cluster, the resolution will be even greater. In M100, we find the slope of the luminosity function to be in agreement with that previously measured for supergiants in nearby galaxies. Several prominent blue clusters and dense regions in associations have been identified. Starlike objects with magnitudes and colors expected for Cepheid variables at the distance of Virgo have been detected in M100, thus demonstrating the feasibility of determining light curves for actual Cepheid variables in the most distant of our target galaxies. Follow-up observations of an adjacent and partially overlapping field in M100 have now begun with the aim of discovering and determining periods for Cepheids in this galaxy, an important step in the HST Key Project on the Extragalactic Distance scale.

Support for this work was provided by NASA through grant number 2227-87A from the Space Telescope Science Institute which is operated by the Association of Universities for Research in Astronomy, Inc., under NASA contract NAS 526555. B. F. M. was supported in part by the Jet Propulsion Laboratory, California Institute of Technology, under the sponsorship of the Astrophysics Division of NASA's Office of Space Science and Applications. W. L. F.'s work on the extragalactic distance scale is supported in part by NSF grant AST 91-16496. This research has made use of the NASA/IPAC Extragalactic Database (NED) which is operated by the Jet Propulsion Laboratory, Caltech, under contract with NASA.

\section{REFERENCES}

Bahcall, J. N. \& Soneira, R. M. 1981, ApJS, 47, 357

de Vaucouleurs, G., de Vaucouleurs, A., \& Corwin, H. G., Jr. 1976, Second Reference Catalogue of Bright Galaxies (Austin: Univ. of Texas)

Freedman, W. L. 1985, ApJ, 299, 74

Freedman, W. L., et al. 1994, ApJ, 427, 628

Fukugita, M., Hogan, C., \& Peebles, P. J. E. 1993, Nature, 366, 309

Gallagher, J. S., et al. 1994, in preparation

Harris, H. C., Baum, W. A., Hunter, D. A., \& Kreidl, T. J. 1991, AJ, 101, 677

Harris, H. C., Hunter, D. A., Baum, W. A., \& Jones, J. H. 1993, AJ, 105, 1196

Holtzmann, J., et al. 1994, WFPC2 Preliminary Status Rept., 1994 March 16

Hughes, S. M. G., et al. 1994, ApJ, 428, 143

Humphreys, R. M. 1987, PASP, 99, 5

Jacoby, G. H., et al. 1992, PASP, 104, 599

Madore, B. F., \& Freedman, W. L. 1991, PASP, 103, 933

Madore, B. F., et al. 1994, in preparation

Pierce, M. J., McClure, R. D., \& Racine, R. 1992, ApJ, 393, 523

\author{
Ratnatunga, K. U., \& Bahcall, J. N. 1985, ApJS, 59, 63 \\ Sandage, A. R. 1993, ApJ, 402, 3 \\ Sandage, A. R., \& Tammann, G. A. 1981, A Revised Shapley-Ames Catalog of \\ Bright Galaxies (Washington, DC: Carnegie Institution) \\ Shanks, T., Tanvir, N. R., Major, J. V., Doel, A. P., Dunlop, C. N., \& Myers, \\ R. M. 1992, MNRAS, 256, 29P \\ Stetson, P. B. 1992, in PASP Conf. Ser., Vol. 25, Astronomical Data Analysis \\ Software \& Systems, I., ed. D. M. Worrall, C. Biemesderfer, \& J. Barnes (San \\ Francisco: ASP), 297 \\ 1994a, PASP, 106, 250 \\ 1994b, Proc. Space Telescope Workshop, Calibrating Hubble Space \\ Telescope (Baltimore: STScI), 89 \\ Tonry, J. L., Ajhar, E. A., \& Luppino, G. A. 1990, AJ, 100, 1416 \\ Tully, R. B. 1988, Nearby Galaxies Catalog (Cambridge: Cambridge Univ. \\ Press)
}

Note added in proof.-As this paper is going to press, we report that Cepheid variables have now been discovered in M100 and a direct distance to this galaxy has been measured (W. L. Freedman et al., Nature, in press [1994]). 


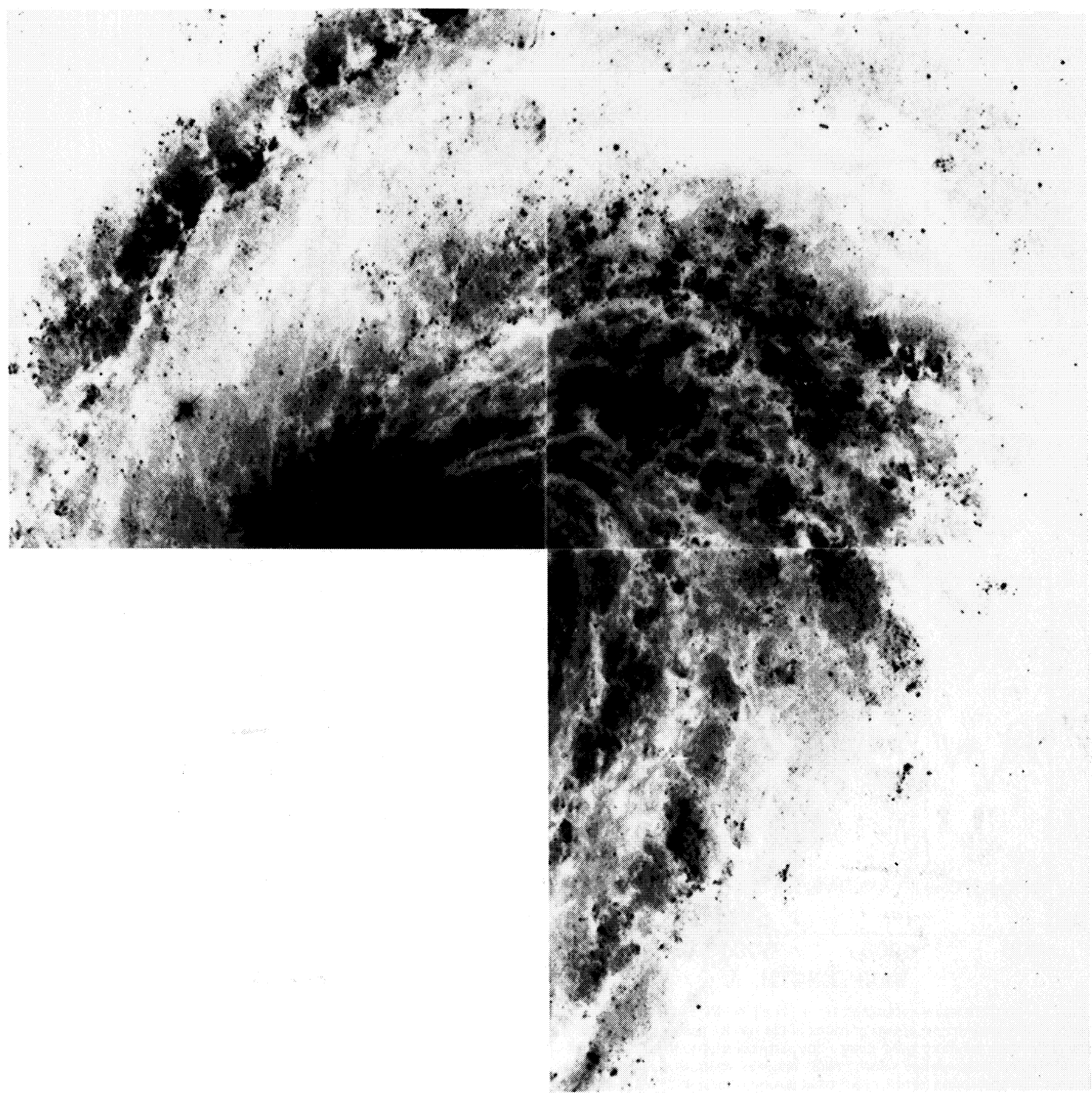

Fig. 1.-A montage of the three WFC F555W images for the observed M100 field

FREEDMAN et al. (see 435, L32) 